Review

\title{
Mechanistic Insights of Actions of Metal Nanoparticles on Multidrug-Resistant Bacteria
}

\author{
${ }^{1,2 *}$ Seham S. Alzahrani, ${ }^{1}$ Hani Mohammed Ali and ${ }^{1,3}$ Mohamed M. Ahmed \\ ${ }^{1}$ Department of Biological Sciences, Faculty of Science, P.O. Box 80203, King Abdelaziz University, Jeddah, 21589, Saudi Arabia \\ ${ }^{2}$ Department of Biotechnology, Faculty of Science, Taif University, Saudi Arabia \\ ${ }^{3}$ Department of Nucleic Acids Research, Genetic Engineering and Biotechnology Research Institute (GEBRI), \\ City for Scientific Research and Technological Applications. Alexandria, Egypt
}

\author{
Article history \\ Received: 10-04-2021 \\ Revised: 08-06-2021 \\ Accepted: 15-06-2021 \\ Corresponding Author: \\ Seham S. Alzahrani \\ Department of Biological \\ Sciences, Faculty of Science, \\ P.O. Box 80203, King \\ Abdelaziz University, Jeddah, \\ 21589, Saudi Arabia \\ Email: seham.alzahrani@hotmail.com
}

\begin{abstract}
Multidrug-Resistant bacteria (MDR) are the most serious threat to public health. The number of infections caused by resistant strains is on the rise all over the world. At this time, new techniques for developing antimicrobial drugs are becoming necessary. Metallic Nanoparticles (MNPs) in the biomedical field are one of the most promising methods to overcome antibiotic resistance. Nanoparticles have recently been used as antiseptics and antimicrobials to treat a variety of diseases, including Gram-positive and Gram-negative bacteria. The disruptions caused by nanoparticles to bacterial cells include changing bacterial cell walls, denaturation of bacterial DNA and inhibition of bacterial replications. These processes are still, however, not completely understood. The DNA damage caused by Nanoparticles (NPs) results from penetrating the bacterial cell membrane due to its small size and large surface area. This action causes bacterial DNA replication to be disrupted. The use of nanoparticles still presents a therapeutic challenge and much further research is needed to solve this. This review covers the latest approaches to developing new nanobiotechnology techniques that may challenge medical practice to fight against bacteria, particularly MDR bacteria. The study also discusses the up-to-date information about the bacterial resistance mechanism against various antimicrobial agents, identifying the mechanism(s) that can be a therapeutic target. It also discusses the genetic background to silver nanoparticles resistance. 1. Introduction. 2. Mechanisms of Multi-Drug Resistance Bacteria. Antibacterial Mechanisms of Nanoparticles. Role of nanoparticles in therapeutics and drug delivery. Bacterial resistance to NPs.1 General resistance mechanisms. Bacterial resistance to silver 6. Conclusion.
\end{abstract}

Keywords: Metal Nanoparticles, Antimicrobial Against, MultidrugResistant, Nano Biotechnology

\section{Introduction}

Multi-drug resistance bacteria have become significant public health problems that raise the need to develop novel bactericidal materials. This acquired resistance of pathogens presents a key challenge for many antimicrobial drugs (Baptista et al., 2018). The antibiotic resistance crisis has been attributed to the overuse and misuse of these medications. Antibiotics exerts selection pressure on bacteria, resulting in their genetic flexibility and the appearance and continuation of antibiotic resistance. Besides, the number of different forms of antibiotic resistance mechanisms has grown over time (Klemm et al., 2018). The genes coding for resistance to antibiotics is not easily lost once evolved in a bacterial population, in addition to the constant development of resistance (Moodley, 2014). Besides resistance mechanisms are developed, it becomes harder to provide a treatment that can effectively combat this multi-drug resistant phenomenon (Livermore, 2003). With the increasingly complex mutations that occur within bacterial species that allow them to develop drugresistance mechanisms, less and less time, funding and effort are channeled into drug development (Ventola, 2015). Antibiotic treatment of bacterial infections is a path that is becoming increasingly hard to achieve. There are some difficulties in administering many antimicrobial drugs because of their low water solubility, cytotoxicity 
to healthy tissues and rapid degradation and clearance in the bloodstream (Ahangari et al., 2013).

Recent advances in nanotechnology offer new prospects to develop novel formulations based on distinct types of Nanoparticles (NPs) with different sizes and shapes and flexible antimicrobial properties. NPs may be a promising way to fight bacteria by acting as drug delivery systems and natural antimicrobial agents (Wang et al., 2017). Several studies have previously demonstrated that nanoparticles and particularly MNPs overcame these issues and facilitated antimicrobial delivery to microbial infection sites (Ahangari et al., 2013).

\section{Mechanisms of Multi-Drug Resistance Bacteria}

All the way through history, there has been a brutal fight between humans and pathogenic microorganisms due to the severe infections caused by pathogens. To overcome the disease of microorganisms, the use of antimicrobial agents came into existence long back. The first and foremost antibacterial agent, penicillin (a specific antimicrobial agent against bacteria), revolutionized the entire world in the 1940s. The widespread use of antimicrobial drugs (penicillin and sulfa drugs) started in the 1950 s. Since then, many antibiotics have been developed and used for the treatment of several diseases. However, the satisfaction of defeating pathogenic microorganisms was shortlived due to the emergence of antimicrobial drug resistance in microorganisms. The ability of microorganisms to resist the effect of a previously used drug to destroy them is known as antimicrobial drug resistance and it is a serious problem in science.

The various drug resistance mechanisms present in bacteria have been discussed below (Carpenter and Chambers, 2004; Zhu et al., 2010):

a) Components of the bacterial envelope (glycocalyx, cell wall, or plasma membrane) can prevent the drug's penetration and entry inside, thus making the bacterial cell resistant to a particular medicine. For example, the attendance of outer membrane in many gram-negative bacteria makes them resistant to various antibiotics such as rifampin, penicillin, etc.

b) Several pathogenic bacteria have not-specific transporter proteins known as "efflux pump" in their plasma membrane, which can expel the drug from a cell and make the bacteria resistant. From the first drug-resistant efflux pump discovered in the 1990s, the development in molecular microbiology has led to the characterization of many efflux pumps in Gram-positive bacteria including Methicillin-Resistant Staphylococcus Aureus (MRSA), Streptococcus pneumoniae, Clostridium difficile, Enterococcus spp. and Listeria monocytogenes and Gram-negative bacteria such as Acinetobacter baumannii, Escherichia coli, Klebsiella pneumoniae, Stenotrophomonas maltophilia, Campylobacter jejuni, Pseudomonas aeruginosa, Neisseria gonorrhoeae, Vibrio cholerae and Salmonella spp (Sharma et al., 2019)

c) Bacteria can develop resistance by causing a structural transformation of the drug being used, rendering it inactive. For example, the enzyme $\beta$ lactamase, which is found in many Staphylococci species, hydrolyzes the $\beta$-lactam ring of penicillin, rendering it inactive and unable to destroy bacteria. Similarly, the acetyltransferase enzyme found in some bacteria makes them resistant to antibiotics, especially chloramphenicol, since it causes acetylation of the amino group present in chloramphenicol, which causes it to deactivate (Domalaon et al., 2018)

d) Bacteria may also develop resistance to a drug by altering their cellular structures (such as the peptide bridge in the cell wall, the ribosome, etc.) as well as the enzymes that the drug targets. Their bacterial metabolism is improved, as a result, making them less susceptible to the antibiotic (Domalaon et al., 2018)

e) Pre-existing genes on the bacterial chromosome or plasmid may also cause antibiotic resistance. A bacterial cell may also acquire these genes by lateral or horizontal gene transfer (Sultan et al., 2018)

\section{Antibacterial Mechanisms of Nanoparticles}

Nanomaterials have at least one measurement in the nanometer scale range $(1-100 \mathrm{~nm})$ that convey particular physical and chemical properties considerably different from those of bulk materials (Wang et al., 2017). Nanoparticles have sparked particular interest among the diverse range of nanomaterials. Nanoparticles have many characteristics that make them perfect as drug delivery vehicles for disease-causing pathogens. Nanoparticles, especially metal nanoparticles, have become increasingly popular in biomedical research in recent years. Because of their unique properties of small scale, large area-tovolume ratio, high reactivity to living cells, hightemperature stability and cell translocation, they are used in bioimaging, drug delivery and other diagnostic and therapeutic applications. Metal nanoparticles have always been used against several diseases; in the past, it found use as an antiseptic and antimicrobial against Gram-positive and Gram-negative bacteria (Halawani, 2016).

Metal nanoparticles have been widely used in a variety of medical applications. Gold Nanoparticles (AuNPs) are widely reported to be guiding an astounding rebound and are particularly exceptional among them. AuNPs have gotten a lot of interest because of their multiple, unique 
functional characteristics and ease of production. Their intrinsic features (optics, electronics and physicochemical characteristics) can be altered by changing the characterization of the nanoparticles, such as shape, size and aspect ratio. Their intrinsic properties (optics, electronics and physicochemical properties) can be adjusted by modifying the nanoparticles' characterisation, such as form, size and aspect ratio (Hu et al., 2020).

Among metal nanoparticles, Zinc Oxide Nanoparticles ( $\mathrm{ZnO} \mathrm{NPs})$ are interesting because to their unique advantages, which include a broad band gap, a high binding energy and a strong piezoelectric property. $\mathrm{ZnO}$
NPs, which can have a wide range of nanostructures, are thought to be biosafe, nontoxic and biocompatible and have been used in a variety of technologies and industries including optoelectronics, piezoelectric and magnetic sensors, biodiagnosis, biological labeling, environmental protection, biology and the pharmaceutical industry (Selim et al., 2020). Zinc oxide nanoparticles are widely known to be antibacterial and to inhibit the growth of microorganisms by penetrating the cell membrane. Oxidative stress causes lipid, carbohydrate, protein and DNA damage (Siddiqi et al., 2018).

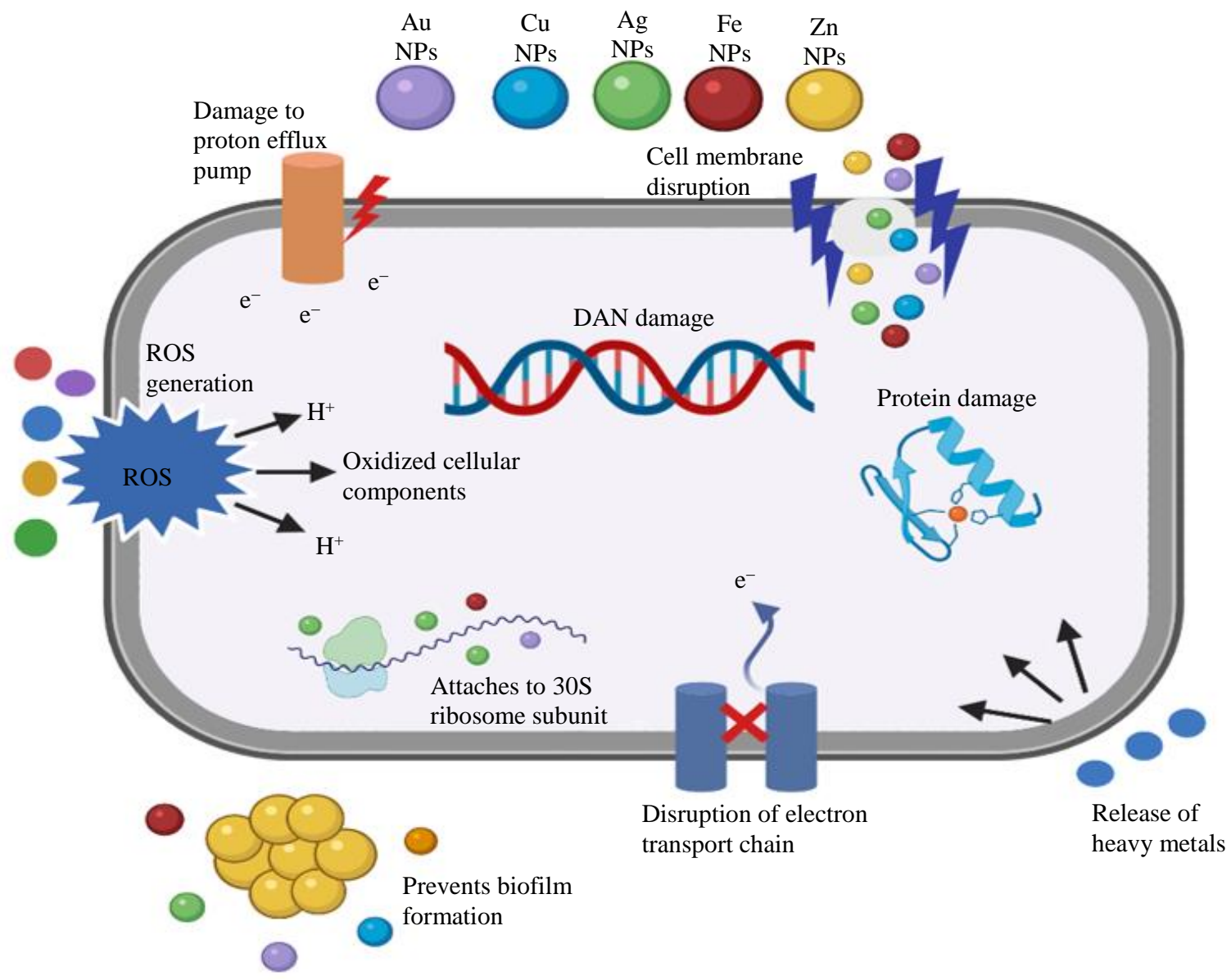

Fig. 1: Different mechanisms of action of Nanoparticles (NPs) in bacterial cells

Table 1: Types of antimicrobial nanomaterials and their antimicrobial mechanisms (Shaikh et al., 2019)

\begin{tabular}{ll}
\hline Nanomaterial & Antimicrobial mechanism \\
\hline Silver & Damages DNA and electron transport; disruption of the cellular membrane. \\
Gold & Heavy electrostatic attraction to cell surfaces; accumulation at and interaction with cell membrane and wall \\
Copper & Oxidative stress and DNA degradation; membrane damage \\
Zinc oxide & Damage to the cell membrane; accumulation inside the cell and production of toxic $\mathrm{H}_{2} \mathrm{O}_{2}$ \\
Titanium dioxide & Damage to cell membranes and walls; production of toxic reactive oxygen species \\
Chitosan & Disruption of cell membranes; inactivation of enzymes; chelation of trace metals \\
Nitric oxide-releasing NPs & Release of nitric oxide and production of reactive oxygen species \\
\hline
\end{tabular}


In the most recent studies, nanomaterial antibacterial activity is attributable to at least one strategy of the following: Inhibition of cell wall and membrane synthesis, generation of toxic Reactive Oxygen Species (ROS), energy transduction disruption, enzyme inhibition and reduced DNA production (Weir et al., 2008). Some welldocumented antimicrobial nanomaterials are described in Table 1 and their probable antibacterial mechanisms are detailed in Fig. 1. In general, NPs interaction with procaryotic and eucaryotic cells depends on size, shape, surface charge, functionalisation and core structure are important factors that determine the biological effects of nanoparticles, such as cellular uptake, cellular activation, as well as intercellular distribution (Helmlinger et al., 2016).

\section{Role of Nanoparticles in Therapeutics and Drug Delivery}

As described above, NPs can fight bacterial and microbial resistance on their own and act as the "medium and carrier" of antibiotics. Nanostructured materials may circumvent drug resistance mechanisms in bacteria and associated with their antimicrobial potential, inhibit biofilm formation or other important processes (Baptista et al., 2018). The mechanisms of drug delivery based on NPs, however, differ from those previously presented. Many types of nanoparticles are presently used for drug delivery (Daeihamed et al., 2017) Solid Lipid (SL) NPs, (Naseri et al., 2015; Kakkar Thukral et al., 2014), polymer-based NPs, polymer mice, inorganic nano-drug carriers (such as magnetic NPs, carbon nanomaterials, mesoporous silica NPs and quantum dots), terpenoid-based NPs, (Abed and Couvreur, 2014) and dendrimer NPs (Liu et al., 2015). In comparison to conventional delivery systems, NPs have the following major advantages.

\section{Size}

Nanoparticles' ultra-small size and controllable size make them ideal for antimicrobial activity and fighting bacteria intracellularly (Ranghar et al., 2014). Treatment of infectious diseases caused by drug-resistant strains is more complicated by antibiotic use (Andrade et al., 2013) due to low membrane antibiotic transport.

\section{Protection}

Carriers of NPs may increase serum antibiotics and protect drugs from the resistance of target bacteria. Drugs are protected from harmful chemical reactions within NPs carriers; therefore, the efficacy of drugs can be maintained.

Precision and security: NPs carriers can target an infection site using antibiotics and decrease systemic side effects. High-dose drug absorption at the target site is challenging to encourage while avoiding side effects (including drug toxicity) when using traditional antibiotics without a carrier. Antibacterial drug delivery systems based on NPs deliver the drug to the site of action while minimizing side effects. Because of the higher dose administered to the infection site, the unwanted side effects of antibiotics on the body are weakened.

Controllability: Antibiotics can be released in a flexible and controlled manner. With a convenient delivery method, the blood drug level is maintained for a short time in a relatively large range that can exceed the maximally tolerated dose or fail to reach the lowest effective dose.

Combination: Multiple drugs or antimicrobials can be packed into a single NPs and NPS can be paired with other structures to enhance the agents' antibacterial properties (Wang et al., 2017).

As other resistance mechanisms are developed, it becomes harder to provide a treatment that can effectively combat this multidrug-resistant phenomenon (Moodley, 2014). Some of the other problematic areas of medical treatment and the solutions offered by nanotechnology are outlined in (Table 2).

Table 2: Challenges and solutions to current diseases (Couvreur and Vauthier, 2006)

\begin{tabular}{lll}
\hline Disease & Therapeutic Challenge & Nanotechnology Solution \\
\hline Infections & Increase the efficacy of drug delivery & Nanoparticles \\
& Reduce toxicity by & Liposomes \\
& Enhancing intracellular penetration & Micelles \\
& Control of biodistribution & PEGylated nanoparticles \\
& & PEGylated liposomes \\
Cancer & Increase efficacy of delivery & Nanoparticles \\
& Reduce toxicity by & Liposomes \\
& Enhancing intracellular penetration & PEGylated nanoparticles \\
Metabolic Disease & Control of biodistribution & PEGylated liposomes \\
& Controlled and sustained release of drug & Nanoparticles \\
Gene Therapy-related diseases & Protection of molecules against degradation & Liposomes \\
& Improvement of mucosal absorption & Cationic nanospheres \\
& Protect DNA against degradation & Cationic liposomes \\
\hline
\end{tabular}




\section{Bacterial Resistance to NPs}

\section{General Resistance Mechanisms}

Resistance to the antimicrobial agent may occur through either the intrinsic or the acquired mechanisms. Acquired resistance can occur in the form of plasmids, transposons and self-replicating extra-chromosomal DNA through either mutation or the acquisition of different types of genetic material. Acquired resistance has been observed in several micro-organisms to a wide range of antibiotics. Intrinsic resistance is a phenotype exhibited before the use of an antimicrobial agent by microorganisms (Sultan et al., 2018).

Nanoparticles and antibiotics have many different modes of action. Nanoparticles tend to target multiple sites in bacterial cells and therefore have broad-spectrum activity. On the other hand, antibiotics target specific bacterial cell sites and have a narrower spectrum of activity. Resistance to nanoparticles may be acquired through mutations in normal cell genes, plasmids, or transposons (Wang et al., 2017).

Because of their ability to pass from plasmid to chromosome and then transmit resistance to daughter cells, transposons and integrons significantly benefit in producing transferable resistance. Transposons and integrons have also been found to possess a number of genetic determinants that encode resistance to heavy metals. Co-resistance phenotypes induced by neighboring genes are likely to be maintained under the selective pressure exerted by one antimicrobial agent. Therefore, exposure of integron-carrying bacteria to residual nanoparticles' concentrations may encourage antibiotic resistance (Partridge et al., 2018).

Chromosomal mutations in against antibiotics have been recognized for decades. However, there have been fewer studies to see whether the mutation confers resistance to biocides. While $\mathrm{Ag}+$ resistance determinants in Enterobacteriaceae have recently been cloned and sequenced, no Gram-positive bacteria have been identified despite the clinical use of silver compounds in Staphylococci and other Gram-positive bacteria (Wales and Davies, 2015).

Silver nanoparticles suggested that widespread and uncontrolled use of $\mathrm{Ag}^{+}$may result in more bacteria developing resistance. However, the possibility of transfer of silver resistance genes is considered low, unstable and difficult to maintain and transfer. The frequency of occurrence of $\mathrm{Ag}+$ resistance is variable, with a poor understanding of the conditions for distinguishing between Ag+ resistance and Ag+ sensitivity. Examples of silver-resistant bacterial strains isolated include $E$. Coli, Enterobacter cloacae, Klebsiella pneumoniae, Acinetobacter baumannii, Salmonella typhimurium, Pseudomonas stutzeri (Franci et al., 2015).

\section{Bacterial Resistance to Silver}

Various silver types have been used as an important antibacterial agent for several years and their antibacterial activity has remained constant. No definitive reports have been produced yet on the production of bacterial resistance to silver NPs (Haefeli et al., 1984). However, several reports of bacterial resistance to ionic silver have surfaced. Reducing silver ionic $\left(\mathrm{Ag}^{+}\right)$to a less toxic neutral oxidation state or active efflux of $\left(\mathrm{Ag}^{+}\right)$from the cell by P-type adenosine triphosphatases or chemiosmotic $\mathrm{Ag}^{+} / \mathrm{H}^{+}$antiporters are two examples of such resistance (Li et al., 1997). Moreover, preliminary evidence of bacterial resistance or reduced susceptibility to silver NPs has been presented in a few recent studies. The majority of these experiments used resistance mechanisms focused on removing silver ionic forms (Graves Jr et al., 2015).

Since the repatriation of silver nanoparticles products as alternatives for treating burn wounds, the number of reports of bacteria resistance to silver has increased. In burn treatment centers, silver-resistant bacteria were mostly isolated from patients and isolated from the environment (Cooper and Kirketerp-Møller, 2018).

In Gram-negative species, efflux has been suggested as an essential mechanism of bacterial resistance. The Sil operon, present in Salmonella typhimurium isolates, is the genetic and molecular basis of silver resistance (Gupta et al., 1999). The operon codes for the silver binding proteins SilE and possibly SilF, the two efflux pumps SilCBA and SilP and the regulatory proteins SilR (Fig. 2). Sil operon was found on plasmids that also carried antibiotic resistance genes (Fang et al., 2016).

In addition to the intense, long-term and repeated administration of classical antibiotics or ionic types of silver, silver NPs have not been widely used in medical practice, except for a brief time at the turn of the twentieth century when colloidal silver was used to treat several diseases (Panáček et al., 2018). As a result, it's unknown if bacteria can gain resistance to silver nanoparticles. Despite these efforts, the issue of whether bacteria will develop resistance to silver NPs antibacterial effect after long-term exposure to subinhibitory silver concentrations is still being debated (Gunawan et al., 2013).

After repeated exposure to silver nanoparticles, Gram-negative bacteria such as E. coli and P. aeruginosa may develop resistance to them. The resistance is caused by the processing of the adhesive protein flagellin, which allows the nanoparticles to agglomerate together. This resistance arises without the need for genetic changes; all that is needed is a phenotypic change to decrease the nanoparticles' colloidal stability and thus eradicate their antibacterial activity. Additional stabilization of silver nanoparticles with surfactants or polymers would not overcome the resistance mechanism (Panáček et al., 2018). 


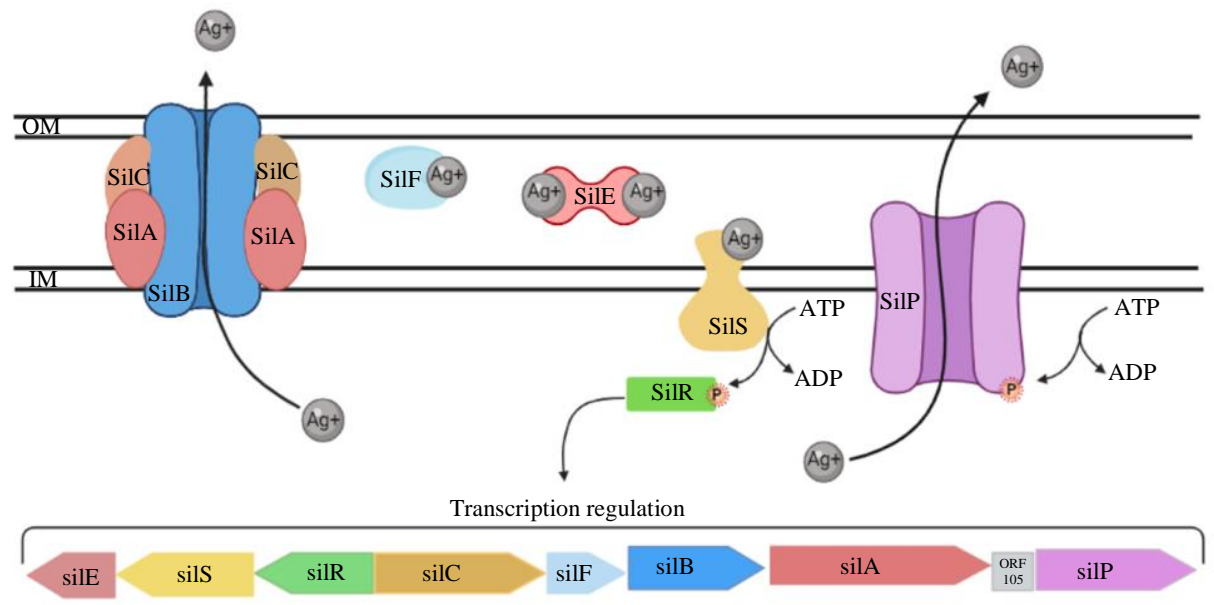

Fig. 2: The Sil operon and its proposed transcriptional products. Top: Proposed function of genes from the Sil operon. Bottom: The Sil operon of plasmid. OM-outer membrane, IM-inner membrane

\section{Conclusion}

This review discusses the mechanism of bacterial resistance against various antimicrobial agents, which will help to identify mechanisms that may be a therapeutic target in the current age of increasing antibiotic resistance. The current, thorough review of antibacterial mechanisms may contribute to the production of useful antibacterial NPs.

\section{Acknowledgment}

The authors acknowledge assistance from the Science and Technology Unit, Deanship of Scientific Research and Deanship of Graduate Studies and, acknowledge support from the Dept. of Biological Sciences, Faculty of Science, King Abdulaziz University (KAU), Jeddah, KSA.

\section{Author's Contributions}

Seham S Alzahrani: Participated in all experiments and contributed to the writing of the manuscript.

Hani Mohammed Ali: Participated in coordinated the data analysis and contributed to the writing of the manuscript.

Mohamed M Ahmed: Designed the research plan and organized the study.

\section{Ethics}

This article is original and contains unpublished material. The corresponding author confirms that all of the other authors have read and approved the manuscript and no ethical issues involved.

\section{References}

Abed, N., \& Couvreur, P. (2014). Nanocarriers for antibiotics: a promising solution to treat intracellular bacterial infections. International Journal of Antimicrobial Agents, 43(6), 485-496. https://doi.org/10.1016/j.ijantimicag.2014.02.009

Ahangari, A., Salouti, M., Heidari, Z., Kazemizadeh, A. R., \& Safari, A. A. (2013). Development of gentamicin-gold nanospheres for antimicrobial drug delivery to Staphylococcal infected foci. Drug Delivery, 20(1), 34-39. https://doi.org/10.3109/10717544.2012.746402

Andrade, F., Rafael, D., Videira, M., Ferreira, D., Sosnik, A., \& Sarmento, B. (2013). Nanotechnology and pulmonary delivery to overcome resistance in infectious diseases. Advanced Drug Delivery Reviews, 65(13-14), 1816-1827. https://doi.org/10.1016/j.addr.2013.07.020

Baptista, P. V., McCusker, M. P., Carvalho, A., Ferreira, D. A., Mohan, N. M., Martins, M., \& Fernandes, A. R. (2018). Nano-strategies to fight multidrug resistant bacteria-"A Battle of the Titans". Frontiers in Microbiology, 9, 1441. https://doi.org/10.3389/fmicb.2018.01441

Carpenter, C. F., \& Chambers, H. F. (2004). Daptomycin: another novel agent for treating infections due to drugresistant gram-positive pathogens. Clinical Infectious Diseases, 38(7), 994-1000. https://doi.org/10.1086/383472

Cooper, R., \& Kirketerp-Møller, K. (2018). Nonantibiotic antimicrobial interventions and antimicrobial stewardship in wound care. Journal of Wound Care, 27(6), 355-377. https://doi.org/10.12968/jowc.2018.27.6.355 
Couvreur, P., \& Vauthier, C. (2006). Nanotechnology: intelligent design to treat complex disease. Pharmaceutical Research, 23(7), 1417-1450. https://doi.org/10.1007/s11095-006-0284-8

Daeihamed, M., Dadashzadeh, S., Haeri, A., \& Faghih Akhlaghi, M. (2017). Potential of liposomes for enhancement of oral drug absorption. Current Drug Delivery, 14(2), 289-303. https://doi.org/10.2174/1567201813666160115125756

Domalaon, R., Idowu, T., Zhanel, G. G., \& Schweizer, F. (2018). Antibiotic hybrids: the next generation of agents and adjuvants against Gram-negative pathogens?. Clinical Microbiology Reviews, 31(2), e00077-17. https://doi.org/10.1128/CMR.00077-17

Fang, L., Li, X., Li, L., Li, S., Liao, X., Sun, J., \& Liu, Y. (2016). Co-spread of metal and antibiotic resistance within ST3-IncHI2 plasmids from E. coli isolates of food-producing animals. Scientific Reports, 6(1), 1-8. https://doi.org/10.1038/srep25312

Franci, G., Falanga, A., Galdiero, S., Palomba, L., Rai, M., Morelli, G., \& Galdiero, M. (2015). Silver nanoparticles as potential antibacterial agents. Molecules, 20(5), 8856-8874.

https://doi.org/10.3390/molecules20058856

Graves Jr, J. L., Tajkarimi, M., Cunningham, Q., Campbell, A., Nonga, H., Harrison, S. H., \& Barrick, J. E. (2015). Rapid evolution of silver nanoparticle resistance in Escherichia coli. Frontiers in Genetics, 6, 42. https://doi.org/10.3389/fgene.2015.00042

Gunawan, C., Teoh, W. Y., Marquis, C. P., \& Amal, R. (2013). Induced adaptation of Bacillus $\mathrm{sp}$. to antimicrobial nanosilver. Small, 9(21), 3554-3560. https://doi.org/10.1002/smll.201300761

Gupta, A., Matsui, K., Lo, J. F., \& Silver, S. (1999). Molecular basis for resistance to silver cations in Salmonella. Nature Medicine, 5(2), 183-188. https://doi.org/10.1038/5545

Haefeli, C., Franklin, C. H. R. I. S. T. O. P. H. E. R., \& Hardy, K. (1984). Plasmid-determined silver resistance in Pseudomonas stutzeri isolated from a silver mine. Journal of Bacteriology, 158(1), 389-392. https://doi.org/10.1128/jb.158.1.389-392.1984

Halawani, E. M. (2016). Nanomedicine opened new horizons for metal nanoparticles to treat multi-drug resistant organisms. International Journal Current Microbiology Application Science, 5, 397-414. https://doi.org/10.20546/ijcmas.2016.502.045

Helmlinger, J., Sengstock, C., Groß-Heitfeld, C., Mayer, C., Schildhauer, T. A., Köller, M., \& Epple, M. (2016). Silver nanoparticles with different size and shape: equal cytotoxicity, but different antibacterial effects. RSC Advances, 6(22), 18490-18501. https://doi.org/10.1039/C5RA27836H
Hu, X., Zhang, Y., Ding, T., Liu, J., \& Zhao, H. (2020). Multifunctional Gold Nanoparticles: A Novel Nanomaterial for Various Medical Applications and Biological Activities. Frontiers in Bioengineering and Biotechnology, $8,990$. https://doi.org/10.3389/fbioe.2020.00990

Kakkar Thukral, D., Dumoga, S., \& K Mishra, A. (2014). Solid lipid nanoparticles: promising therapeutic nanocarriers for drug delivery. Current Drug Delivery, 11(6), 771-791. https://doi.org/10.2174/156720181106141202122335

Klemm, E. J., Wong, V. K., \& Dougan, G. (2018). Emergence of dominant multidrug-resistant bacterial clades: Lessons from history and whole-genome sequencing. Proceedings of the National Academy of Sciences, 115(51), 12872-12877. https://doi.org/10.1073/pnas.1717162115

Li, X. Z., Nikaido, H., \& Williams, K. E. (1997). Silver-resistant mutants of Escherichia coli display active efflux of $\mathrm{Ag}+$ and are deficient in porins. Journal of Bacteriology, 179(19), 6127-6132. https://doi.org/10.1128/jb.179.19.6127-6132.1997

Liu, Y., K Tee, J., \& NC Chiu, G. (2015). Dendrimers in oral drug delivery application: current explorations, toxicity issues and strategies for improvement. Current Pharmaceutical Design, 21(19), 2629-2642. https://doi.org/10.3109/10717544.2014.895069

Livermore, D. M. (2003). Bacterial resistance: origins, epidemiology and impact. Clinical Infectious Diseases, 36(Supplement_1), S11-S23. https://doi.org/10.1086/344654

Moodley, N. (2014). Antimicrobial activity of ciprofloxacin-coated gold nanoparticles on selected pathogens (Doctoral dissertation). https://www.semanticscholar.org/paper/Antimicrobi al-activity-of-ciprofloxacin-coated-goldMoodley/2af15df127049cac53fa4f087c009444828d b0da.

Naseri, N., Valizadeh, H., \& Zakeri-Milani, P. (2015). Solid lipid nanoparticles and nanostructured lipid carriers: structure, preparation and application. Advanced Pharmaceutical Bulletin, 5(3), 305. https://doi.org/10.15171/apb.2015.043

Panáček, A., Kvítek, L., Smékalová, M., Večeřová, R., Kolár̆, M., Röderová, M., ... \& Zbořil, R. (2018). Bacterial resistance to silver nanoparticles and how to overcome it. Nature Nanotechnology, 13(1), 65-71. https://doi.org/10.1038/s41565-017-0013-y

Partridge, S. R., Kwong, S. M., Firth, N., \& Jensen, S. O. (2018). Mobile genetic elements associated with antimicrobial resistance. Clinical Microbiology Reviews, 31(4), e00088-17. https://doi.org/10.1128/CMR.00088-17 
Ranghar, S., Sirohi, P., Verma, P., \& Agarwal, V. (2014). Nanoparticle-based drug delivery systems: promising approaches against infections. Brazilian Archives of Biology and Technology, 57(2), 209-222. https://doi.org/10.1590/S1516-89132013005000011

Selim, Y. A., Azb, M. A., Ragab, I., \& Abd El-Azim, M. H. (2020). Green synthesis of zinc oxide nanoparticles using aqueous extract of Deverra Tortuosa and their cytotoxic activities. Scientific reports, 10(1), 1-9. https://doi.org/10.1038/s41598020-60541-1

Shaikh, S., Nazam, N., Rizvi, S. M. D., Ahmad, K., Baig, M. H., Lee, E. J., \& Choi, I. (2019). Mechanistic insights into the antimicrobial actions of metallic nanoparticles and their implications for multidrug resistance. International Journal of Molecular Sciences, 20(10), 2468. https://doi.org/10.3390/ijms20102468

Sharma, A., Gupta, V. K., \& Pathania, R. (2019). Efflux pump inhibitors for bacterial pathogens: From bench to bedside. The Indian Journal of Medical Research, 149(2), 129. https://doi.org/10.4103/ijmr.IJMR_2079_17

Siddiqi, K. S., ur Rahman, A., \& Husen, A. (2018). Properties of zinc oxide nanoparticles and their activity against microbes. Nanoscale research letters, 13(1), 1-13. https://doi.org/10.1186/s11671-018-2532-3
Sultan, I., Rahman, S., Jan, A. T., Siddiqui, M. T., Mondal, A. H., \& Haq, Q. M. R. (2018). Antibiotics, resistome and resistance mechanisms: A bacterial perspective. Frontiers in microbiology, 9, 2066. https://doi.org/10.3389/fmicb.2018.02066

Ventola, C. L. (2015). The antibiotic resistance crisis: part 1: causes and threats. Pharmacy and Therapeutics, 40(4), 277.

Wales, A. D., \& Davies, R. H. (2015). Co-selection of resistance to antibiotics, biocides and heavy metals and its relevance to foodborne pathogens. Antibiotics, 4(4), 567-604. https://doi.org/10.3390/antibiotics4040567

Wang, L., Hu, C., \& Shao, L. (2017). The antimicrobial activity of nanoparticles: present situation and prospects for the future. International Journal of Nanomedicine, 12, 1227. https://doi.org/10.2147/IJN.S121956

Weir, E., Lawlor, A., Whelan, A., \& Regan, F. (2008). The use of nanoparticles in anti-microbial materials and their characterization. Analyst, 133(7), 835-845. https://doi.org/10.1039/b715532h

Zhu, L., Lin, J., Ma, J., Cronan, J. E., \& Wang, H. (2010). Triclosan resistance of Pseudomonas aeruginosa PAO1 is due to FabV, a triclosan-resistant enoylacyl carrier protein reductase. Antimicrobial Agents and Chemotherapy, 54(2), 689-698. https://doi.org/10.1128/AAC.01152-09 